\title{
IN-VITRO CULTURE OF OPHIOCORDYCEPS SINENSIS (YARSAGUMBA) AND THEIR ASSOCIATED ENDOPHYTIC FUNGI OF NEPAL HIMALAYA
}

\author{
Bikash Baral* and Jyoti Maharjan* \\ *Nepal Academy of Science and Technology (NAST).
}

\begin{abstract}
An array of Ophiocordyceps sinensis and its associated endophytic fungi residing in the high Nepalese meadows were isolated and studied for their association and symbiotic effectiveness with the host plant. Fungi are noted to be quite common in nature and some of them have been shown to have adverse effects against insects, nematodes and plant pathogens. Out of the few isolates, different types of associated fungi with Ophiocordyceps sinensis were screened viz. Acremonium strictum, Altenaria solani, Aspergillus flavus, Trichoderma virens, Colletotrichum gloeosporiode, Curvularia lunata, Thielaviopsis sp. and Verticillium sp. The growth rate of the Ophiocordyceps sinensis was found to be very slow as compared to other associated fungi with the diameter of only $1 \mathrm{~cm}$ in 2 weeks interval. Fast growing endophytic fungi was found to be Acremonium strictum followed by Altenaria solani. However, the maintenance of the pure culture of the Ophiocordyceps fungi is rather difficult and was soon covered up with other associated fungi. Besides, it was rather difficult to sporulate the $O$. sinensis in the laboratory conditions despite using specific medias.
\end{abstract}

Keywords: Caterpillar mushroom; Endophytic fungi; Insect-fungi combination; In-vitro culture; Sporulation.

\section{INTRODUCTION}

Ophiocordyceps sinensis (syn. Cordyceps sinensis) (Berk.) G. H. Sung, J. M. Sung, Hywel-Jones \& Spatafora, a caterpillar fungus (Winkler, 2008a) is an entomopathogenic fungi (Hajek and Leger, 1994; Kim et al., 2003) belonging to the order Hypocreales of the family Clavicipitaceae (Sung et al., 2001) infecting and eventually killing the mummified Lepidopteran larvae (Hajek and Leger, 1994) usually that of the Himalayan Bat Moth, Hepialus armonicanus (Holliday et al., 2005). Cordyceps are insect parasitic fungi, exhibiting a high degree of host specificity (Paterson, 2008). A high degree of genetic variation within Cordyceps sinensis exist because of the Lepidopterean host that do not represent a monophyletic group, finally creating difficulties in verifying samples. In nature, it is found only at high alps at an altitude ranging from 4,600 -5,000m asl (Garbyal et al., 2004; Winkler, 2008a), appearing annually on the Himalayan Plateau of Nepal, Sikkim, Bhutan, Arunchal Pradesh (Negi et al., 2006), and Tibetan plateau (Garbyal et al., 2004), and because of being very small and their growth restricted to a small area (Kim et al., 2003), they are thus very difficult to harvest (Holliday et al., 2005). The fungus is endemic in the alpine shrub-meadow zone of high mountains or highlands of Northern Nepal (Zang and Kinjo, 1998). About 400 species of Cordyceps are known so far (Shimizu, 1994), and they are classified by color and shape of fruiting body or spore, shape of the ascus, and kind of the infecting host insect (Shimizu,
1994; Ito \& Hirano, 1997).The caterpillar, being 3.5-4 cm long, is usually yellowish when cleaned while the stroma, usually 4-10 cm, is dark brown or black. Cordyceps species are mainly found in the Dolpa district of Nepal and the other important reported districts are Darchula, Bajhang, Mugu, Humla and Rasuwa. The main harvesting period ranges from May-July.

Kobayashi (1941) listed 137 species in the genus Cordyceps and, of these, 125 were parasitic on insects (with one or two growing on subterrarean insects), Dube (1983) identified 200 species (Negi et al., 2006) while 400 species of Cordyceps are known so far till date (Shimizu, 1994). The identification of the host insects by larvae stage is rather difficult and identification of the exact host species awaits future determination (Kinjo \& Zang, 2001). The fungus has been noted for its great potential medicinal uses (Winkler, 2008b) and hence extensive pharmaceutical studies have been made (Kinjo et al., 1996).

Ophiocordyceps contain a broad range of bioactive compounds (Kneifel et al., 1977; Furuya et al., 1983), which are considered nutritional (Hobbs, 1995; Holliday and Cleaver, 2004), such as Cordycepin and Cordycepic acid (Chen et al., 1996) with their diverse structural architecture. Others components found included are various saccharides and polysaccharides, including cyclofurans, beta-glucans, beta-mannans, complex polysaccharides etc (Kiho et al., 1996; Bok et al., 1999), anti-bacterial and anti-tumor adenosine derivatives, Ophiocordin, an anti-fungal agent and L-

Author for Correspondence: Bikash Baral, Nepal Academy of Science and Technology (NAST). Email: bikubaral@yahoo.com. 


\begin{tabular}{lccccc}
\hline \hline Fungal strains & \multicolumn{5}{c}{ Growth size (cm) of colony at different days interval* } \\
\cline { 2 - 6 } & $\mathbf{4}$ & $\mathbf{7}$ & $\mathbf{1 0}$ & $\mathbf{1 3}$ & $\mathbf{1 5}$ \\
\hline Acremonium strictum & $1.2 \pm 0.21$ & $1.8 \pm 0.29$ & $2.5 \pm 0.67$ & $3.1 \pm 0.83$ & $3.9 \pm 0.71$ \\
Altenaria solani & $0.9 \pm 0.12$ & $1.6 \pm 0.19$ & $1.9 \pm 0.68$ & $2.6 \pm 0.51$ & $3.6 \pm 0.77$ \\
Aspergillus flavus & $0.8 \pm 0.63$ & $1.4 \pm 0.12$ & $1.4 \pm 0.42$ & $1.9 \pm 0.77$ & $2.9 \pm 0.39$ \\
Trichoderma virens & $3.8 \pm 0.22$ & $5.1 \pm 0.16$ & $6.7 \pm 0.31$ & $10.3 \pm 0.29$ & $13.9 \pm 0.74$ \\
Colletotrichum gloeosporiode & $0.9 \pm 0.6$ & $1.2 \pm 0.14$ & $1.6 \pm 0.23$ & $2.1 \pm 0.3$ & $2.6 \pm 0.14$ \\
Cordyceps sp. & $0.2 \pm 0.16$ & $0.3 \pm 0.07$ & $0.7 \pm 0.31$ & $0.9 \pm 0.37$ & $1.0 \pm 0.41$ \\
Curvularia lunata & $0.9 \pm 0.46$ & $1.6 \pm 0.63$ & $1.7 \pm 0.33$ & $2.5 \pm 0.91$ & $2.7 \pm 0.83$ \\
Thielaviopsis sp. & $0.5 \pm 0.27$ & $1.1 \pm 0.46$ & $1.6 \pm 0.83$ & $1.6 \pm 0.54$ & $2.1 \pm 0.76$ \\
Verticillium sp. & $1.1 \pm 0.23$ & $1.3 \pm 0.36$ & $1.6 \pm 0.19$ & $1.7 \pm 0.36$ & $2.2 \pm 0.10$ \\
\hline$* \bar{x} \pm S D(n=4)$ & & & & &
\end{tabular}

Tryptophan (Paterson, 2008). The caterpillar fungus has a hypoglycemic effect and may be beneficial for people with insulin resistance (Kiho et al., 1993, 1996; Zhao et al., 2002; Lo et al., 2004; Li et al., 2006).

Mycelium of $O$. sinensis has similar clinical efficacy as the natural one and also possess less associated toxicity (Bensky et al., 2004). Optimization of the necessary applicable protocol for laboratory production of the mycelium of $O$. sinensis may prove successful in the preparation of various products from the dried mycelium, which has numerous potential therapeutic applications, as used in the traditional folk medicinal systems. Infact, $O$. sinensis is an intimate relationship of a complex structure of plant and the animal. It is believed that the fungus incorporates some of the insect DNA into its DNA structure creating this type of unparalleled medicinal mushroom. For the formation of the different bioactive compounds, the endophytes residing within the plants have a pivotal role. The aim of the present study was first to culture the Cordyceps specimens in different cultural media and isolating the different associated fungus residing with and within the Cordyceps species.

Different strains of Cordyceps isolated from different places may produce variable cultural characteristics. The extracts of Cordyceps sp. possess extensive pharmacological properties, such as anti-inflammatory, anti-tumor growth, anti-fibrotic and anti-oxidant activities. The size, shape and the color of the colony may differ from the different strains when observed on the artificial solid media and do not change with age. Because of the rarity and peculiarity, fruiting bodies are difficult to form, raising the question of the culture mycelium of being the truly anamorphs of Cordyceps sp.

\section{MATERIALS AND METHODS}

Investigated locality: The samples were harvested with the necessary precautions from the various localities, viz. Manaslu Conservation Area, Manang district and Dolpa district.
Culture Media: All culture medias used viz., Potato Dextrose agar (PDA), Corn Meal agar (CDA), Malt extract agar (MEA), Oat meal agar (OAT), Water agar (WA) were obtained from the HiMedia Pvt. Ltd, India.

Isolation of the fungus: The fungus along with the insect were sealed and harvested from the uprooted site and brought with care to the NAST laboratory. The fungal part was washed carefully with the sterile double distilled deionized water and the head of the stroma was dissected longitudinally. The bundled asci were picked up on the tip of a fine needle and transferred and spread to the surface of a Sabouraud glucose agar plate (SGA; Kinjo \& Zang, 2001). Primary isolation was made after confirmation of germination with the aid of microscope. Germinating single ascospore was picked up and transferred to a fresh SGA slant. The cultures were maintained at $18-20^{\circ} \mathrm{C}$ on the SGA slants at every three days interval starting from four days of and were grown for few weeks for recording its growth requirements.

Morphological Observations: The morphology of the colonies was observed on the different medias (as far as possible selective media, Sabouraud glucose agar, SGA, was used). Petri dishes of $9 \mathrm{~cm}$ diameter containing $20 \mathrm{ml}$ of media were inoculated with $6 \mathrm{~mm}$ disk of mycelia taken from the rim of 30 days old cultures. Plates were kept wrapped in Parafilm to avoid dehydration of the culture media and maintained in the dark, at $24 \pm 1$ ÚC. The growth of the fungal colonies was recorded every three days along two preset diametrical lines. The different stages of the obtained fungus were taken for the observation of its morphological characters. The change in the color of the fungus (if any) was recorded at every alternate day along with their growth rate.

Isolation of the associated fungus: The different fungi in association with the Ophiocordyceps sp. were isolated in the various media viz., Potato Dextrose agar (PDA), Corn Meal agar (CDA), Malt extract agar (MEA), Oat meal agar (OAT), Water agar (WA) etc. The obtained fungi were then isolated by their reconstitution in the respective media till the emergence of pure culture. They were then cover slip cultured and was left to grow on the media for the additional 


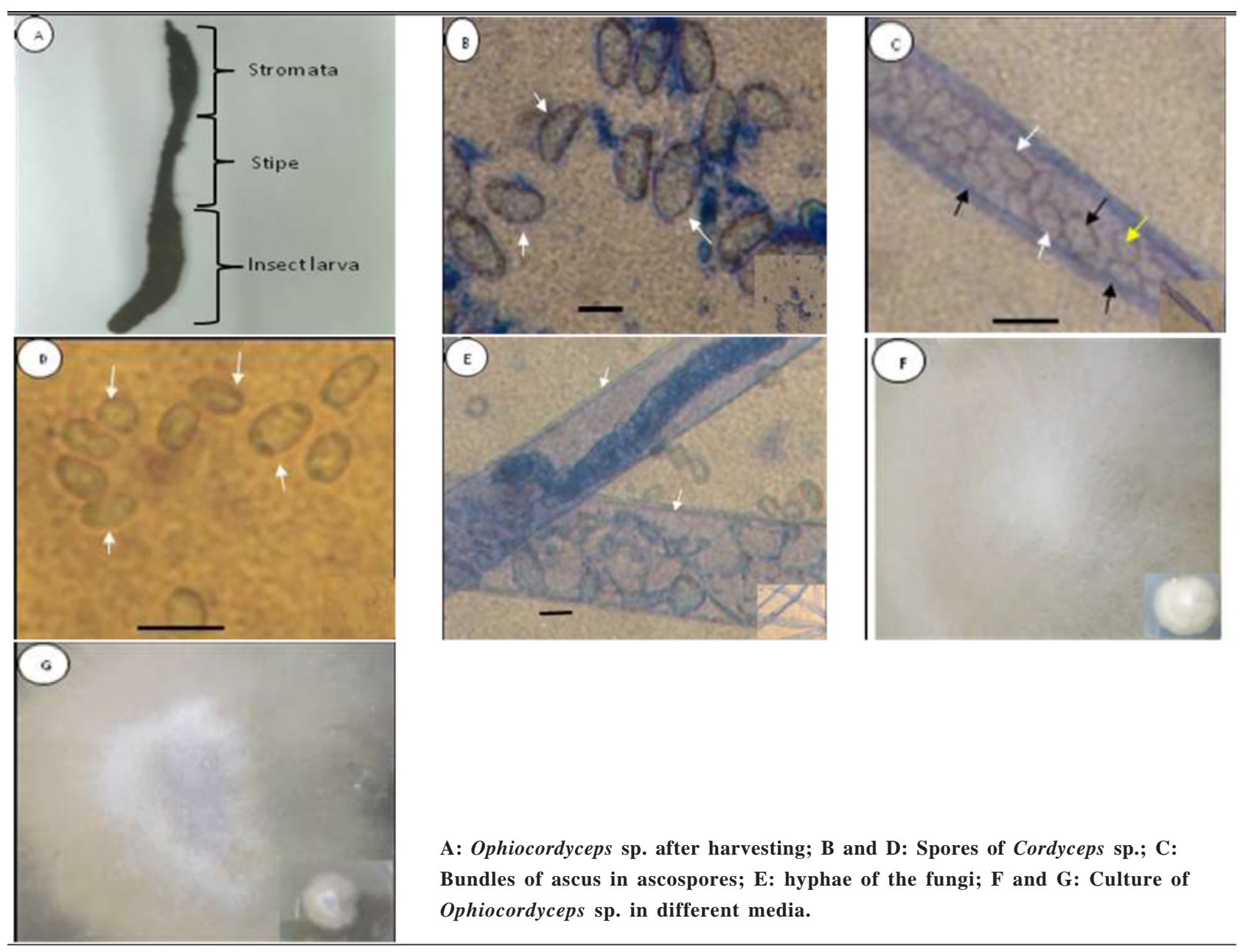

few days till the full growth of the colony. They were followed by mounting on the slides through cellophane technique and staining by cotton blue. The isolates were identified by their colony characters, spores size and shape observing under the compound microscope. The hyphal growths were measured in the peripheral growth zone of the mycelium (Iotti et al., 2005).

\section{Results}

The growth of the $O$. sinensis mycelium was very less as compared to the other associated fungi which was approximately one $\mathrm{cm}$ in two weeks interval time.

Habitat of $\boldsymbol{O}$. sinensis: The collecting sites were high mountain areas, over 5,000m asl, with plains of alpine meadow subdominant with graminea grasses, smart weeds and other herbaceous plants. When the fungus is growing up from the ground after snow melts, it is easy to find the growing stipe protruding from the fruiting body. Below the growing fungus, a small hole approximately $16 \mathrm{~cm}$ deep by $3 \mathrm{~cm}$ wide was usually found. These pores (small holes) were considered the remains of tunnels through which worms which may serve as fungal hosts attacked the roots or rhizomes of several herbaceous plants. These species usually grow on the open high places at the alpine zone where the environmental condition is frigid cold and the oxygen concentration is extremely low. In such harsh conditions where other species fail to grow, Cordyceps is found growing luxuriantly.
Isolation of the associated fungus: The associated fungus along with the $O$. sinensis were Colletotrichum gloeosporiode, Verticillium sp., Aspergillus flavus, Altenaria solani, Acremonium strictum, Trichoderma virens, Curvularia lunata and Thielaviopsis sp.

Morphological and growth characteristics: The morphological and the growth characteristics of the different associated fungus along with the $O$. sinensis were recorded.

\section{Morphological and microscopic characteristics of the associated fungi:}

Colletotrichum gloeosporiode: The fungus produces hyaline, one-celled, ovoid to oblong, slightly curved or dumbbell shaped conidia. Conidia in aggregates seem to be pink in color. Conidiophores simple, elongate; conidia hyaline, 1-celled, ovoid or oblong and parasitic.

Verticillium sp.: Conidiophores are usually well differentiated and erect slender, branched, at least some of the branches verticillate; conidia ovoid to ellipsoid, hyaline, 1-celled, borne singly or in small clusters apically; parasitic on other fungi, or growing saprophytically, fast growing colonies, pale yellow in color, becoming pinkish brown, with a colorless, verticillately branched over most of their length, conidia are hyaline or brightly colored, mostly one-celled.

Aspergillus flavus: Conidiophores upright, simple, terminating in a globose or clavate swelling, bearing phialides 
at the apex or radiating from the entire surface; conidia 1celled, globose, often variously colored in mass ranging from dark black to greenish brown, catenulate, produced basipetally. A large genus containing many species saprophytic on a wide variety of substrata and a few parasitic species.

Altenaria solani: Conidiophores dark, simple, rather short or elongate, typically bearing a simple or branched chain of conidia; conidia dark, typically with both cross and longitudinal septa; variously shaped, obclavate to elliptical or ovoid, frequently born in long chains often more than 4 conidia, less often borne singly and having an apical simple or branched appendage. Conidia pale brown to light brown, ellipsoid, short beak at the tip with surface smooth; produced in chains.

Thielaviopsis sp.: Chlamydospores are produced in chains (two to six spores per chain). The dark, thick walled chlamydospores separate at maturity and each cell is capable of germination. The spores are variable in size with slightly rounded ends. Mycelium is light brown to light black. The septate hyphae are hyaline at first and become pigmented with age.

Curvularia lunata: Hyphae septate; brown, conidia are transversely septate from edge to edge of the conidial wall and are slightly curved.

Acremonium strictum: Slow growing; hyphae are delicate, fine and hyaline; conidia globose to cylindrical and are mostly one-celled.

Trichoderma virens: Cultures were typically fast growing. Colonies were white on potato dextrose agar (PDA). Myceliums were not typically obvious. A yellow pigment may be secreted into the agar, especially on PDA. Conidiophores are highly branched and thus difficult to define or measure, loosely or compactly tufted, often formed in distinct concentric rings or borne along the scant aerial hyphae.

\section{DISCUSSION AND CONCLUSION}

$O$. sinensis is a fungus with a peculiar habitat from the alpine regions of the Tibetan plateau. Due to the herb's rarity, high efficacy, potency in curing various diseases and high prices associated with the wild collected variety, different attempts have long been made to cultivate Ophiocordyceps. These have been made come true by the application of the modern biotechnological - based cultivation methods. Cordyceps species are known to be parasitic on many kinds of insects, and host selectivity is very severe. It is possible that a new classification of Cordyceps species could be constructed according to accumulated phylogenetic information obtained from rDNA sequences (Ito \& Hirano, 1997).

Both $O$. sinensis and its host insects are endemic species on the Himalaya; and the geography and climate may have played an important role in their evolution and coevolution. The differences in the micro-climatic conditions and the life cycle of fungus that parasitizes the insect may also have impacted on the diversification of $O$. sinensis. The caterpillar fungus is more valuable when young, that is, before it sporulates or early during sporulation. In the final stages of sporulation, the host larva becomes soft and undesirable, and the upper part of the mushroom sometimes split. The price of a specimen depends mostly upon the size of the larval host (Winkler, 2008a). The native type specimen of the Nepalese Cordyceps specimens is not available in the national herbarium, questioning about its existence. Many collections of $O$. sinensis by different people are often immature or lack the ascostromatal parts. Therefore, scientific studies concerning this fungus have frequently been confronted with taxonomical problems (Kinjo \& Zang, 2001). There is also a need to evaluate the effectiveness of the fungi, whether the high medicinal properties of the $O$. sinensis is due to its own effect or due to the secretion of the secondary metabolites by the associated endophytic fungi. A question therefore arises, does the fungus itself possess the medicinal properties or are the bioactive compounds being secreted by the combined action of endophytes residing within their cells?

Endemic nature of the fungus along with mass collection attributes from the wild puts it in the category of threatened plants. There is also a need for scientific exploration for the presence of the fungus in different parts of the Himalayan Region, document the occurrence and status, of the wild population and formulate a strategy for conservation as well as sustainable harvesting of the fungus. Hence, it may be concluded that there are many associated species of fungus with the $O$. sinensis which contributes mutually through the secretion of secondary metabolites that possesses high medicinal properties creating this unique unparalled fungi.

\section{ACKNOWLEDGEMENTS:}

The authors are thankful to the scientists of NAST who helped them directly or indirectly with the research solutions during the turbulent times. Thanks are also due to NAST for providing the necessary laboratory facilities. Authors further accolade thanks to Bhusan Shrestha for his critical suggestions and providing the necessary literatures regarding the experiment.

\section{REFERENCES:}

Adhikary, M. K. 2000. Mushrooms of Nepal (ed. Prof. Dr. Durrieu, G.). pp 152-153, 189-190.

Bensky, D., Gamble, A., Clavey, S., Stöger, E. and Bensky, L. L. 2004. Materia Medica: Chinese Herbal Medicine (3rd ed.). Seattle, Washington: Eastland Press. ISBN 978-0-939616-42-8.

Bok, J. W., Lermer, L., Chilton, J., Klingeman, H. G. and Towers, G. H. 1999. Phytochemistry. 51: 891-898.

Chen, S. Z. and Chu, J. Z. 1996. NMR and IR studies on the characterization of cordycepin and 2'deoxyadenosine. Zhongguo Kangshengsu Zaxhi. 21: 9-12.

Dube, H. C. 1983. An Introduction to Fungi. New Delhi: Vikas Publishing House Pvt. Ltd. 616.

Furuya, T., Hirotani, M. and Matsuzawa, M. 1983. N6-(2Hydroxyethyl)adenosine, a biologically active compound from cultured mycelia of Cordyceps and Isaria species. Phytochemistry. 22(11): 2509-2512.

Garbyal, S.S., Agarwal, K.K. and Babu, C.R. 2004. Impact of Cordyceps sinensis in the rural economy of interior villages of Dharchula sub- division of Kumaom Himalayas and its implications 
in the society, Indian Journal of Traditional Knowledge. 3(2): 182 186.

Hajek, A.E. and St. Leger, R.J. 1994. Interactions between fungal pathogens and insect hosts. Annual review of Entomology. 39: 293322.

Hobbs, C. 1995. Medicinal mushrooms: an exploration of tradition, healing, \& culture. 2nd edition. Santa Cruz CA, USA. Botanica Press. 252.

Holliday, J. and Cleaver, M. 2004. On the Trail of the Yak: Ancient Cordyceps in the modern world. http//alohamedicinals.com.

Holliday, J., Cleaver, M. and Wasser, S.P. 2005. Cordyceps. In: Coates PM, Blackman MR, Cragg G, Levine M, Moss J, White J., editors. Encyclopedia of dietary supplements. New York, Marcel Dekker. 1-13.

Iotti, M., Barbieri, E., Stocchi, V. and Zambonelli, A. 2005. Morphological and molecular characterization of mycelia of ectomycorrhizal fungi in pure culture. Fungal Diversity. 19: 51-68.

Ito, Y. and Hirano, T. 1996. First successful amplification of 18 S ribosomal DNA of Cordyceps spp. by the PCR method. Mycoscience. 37: $109-110$.

Ito, Y. and Hirano, T. 1997. The determination of the partial $18 \mathrm{~S}$ ribosomal DNA sequences of Cordyceps species. Lett. Appl. Microbiol. 25: 239-242.

IUCN Nepal. 2000. National Register of Medicinal Plants. Kathmandu, IUCN Nepal. p 158.

Kiho T., Yamane A., Hui, J., Usui, S. and Ukai, S. 1996. Polysaccharides in fungi. XXXVI. Hypoglycemic activity of a polysaccharide (CS-F30) from the cultural mycelium of Cordyceps sinensis and its effect on glucose metabolism in mouse liver. Biological and Pharmaceutical Bulletin. 19 (2): 294-6.

Kiho T., Hui, J., Yamane, A. and Ukai, S. 1993. Polysaccharides in fungi. XXXII. Hypoglycemic activity and chemical properties of a polysaccharide from the cultural mycelium of Cordyceps sinensis. Biological and Pharmaceutical Bulletin. 16 (12): 1291-3.

Kim, K. M., Kwon, Y. G., Chung, H. T., Yun, Y. G., Pae, H. O., Han, J. A., Ha, K. S. and Kim, Y. M. 2003. Methanol extract of Cordyceps pruinosa inhibits in-vitro and in-vivo inflammatory mediators by suppressing NF-kB activation. Toxicol. Appl. Pharmacol. 190: 1-8.

Kinjo, N. and Nian-Lai, H. 1996. Effects of the mycelial extract of cultured Cordyceps sinensis on in vivo hepatic energy metabolism in the mouse. Jpn. J. Pharmacol. 70: 85-88.

Kinjo, N. and Zang, M. 2001. Morphological and phylogenetic studies on Cordyceps sinensis distributed in Southwestern China. Mycoscience 42: 567-574.

Kinjo, N., Nian-Lai, H. and Miyamoto, H. 1996. Effects of the mycelial extract of cultured Cordyceps sinensis on in vivo hepatic energy metabolism in the mouse. Jpn. J. Pharmacol, 70 (1): 85-88.

Kneifel, H., Konig, W.A., Loeffler, W. and Muller, R. 1977. Ophiocordin, an antifungal antibiotic of Cordyceps ophioglossoides. Arch. Microbiol. 113: 121-130.

Kobayasi, Y. 1941. The Genus Cordyceps and its allies. Sci Rep Tokyo Bunrika Daigaku B. 84 (5): 53-260.
Li, S. P, Zhang, G. H. and Zeng, Q. 2006. Hypoglycemic activity of polysaccharide, with antioxidation, isolated from cultured Cordyceps mycelia. Phytomedicine. 13 (6): 428-433.

Lo, H. C., Tu, S. T., Lin, K. C., Lin, S. C. 2004. The antihyperglycemic activity of the fruiting body of Cordyceps in diabetic rats induced by nicotinamide and streptozotocin. Life Sciences. 74 (23): 2897-2908.

Medicinal Plants of Nepal. 1993. H.M.G. of Nepal Ministry of Forests and Soil Conservation, Department of Medicinal Plants, Thapathali, Kathmandu, Nepal, p 116.

Mizuno, T. 1999. Medicinal effects and utilization of Cordyceps (Fr.) Link (Ascomycetes) and Isaria Fr. (Mitosporic Fungi) Chinese Caterpillar Fungi, "Tochukaso" (Review). International Journal of Medicinal Mushrooms. 1 (3): 251-261.

Negi, C. S., Koranga, P. R. and Ghinga, H. S. 2006. Yar tsa gumba (Cordyceps sinensis): A call for its sustainable exploitation. International Journal of Sustainable Development and World Ecology. 13: 1-8.

Paterson, R. M. 2008. Cordyceps - A traditional Chinese medicine and another fungal therapeutic biofactory? Phytochemistry. 69: 14691495.

Shimizu, D. 1994. Color Iconography of vegetable wasps and plant worms. Seibundoshinksosha, Tokyo, 381 pp.

Shrestha, R. and Bhandary, H. 1997. Study on Exploitation of Cordyceps sinensis. Research Center for Applied Science and Technology, Tribhuvan University, Kirtipur, Kathmandu, Nepal.

Spatafora, J. W. and Blackwell, M. 1993. Molecular systematic of unitunicate perithecial ascomycetes: the Clavicipitales-Hypocreales connection. Mycologia. 85: 912-922.

Sung, G. H., Sung, J. M., Hywel-Jones, N.L., Luangsa-ard, J.J., Shrestha, B. and Spatafora, J.W. 2007. MycoBank. International Mycological Association. Retrieved 2011-07-19.

Sung, G., Spatafora, J. W., Zare, R., Hodge, K. T. and Gams, W. 2001. A revision of Verticillium sect. Prostrata. II. Phylogenetic analyses of SSU and LSU nuclear rDNA sequences from anamorphs and teleomorphs of the Clavicipitaceae. Nova Hedwigia. 72: 311328.

Wang, S. and Shiao, M. S. 2000. Pharmacological functions of Chinese Medicinal Fungus Cordyceps sinensis and related species. Journal of Food and Drug Analysis. 8 (4): 248-257.

Winkler, D. 2008a. The mushrooming fungi market in Tibet exemplified by Cordyceps sinensis and Tricholoma matsutake. Journal of the International Association of Tibetan Studies. In: In the Shadow of the Leaping Dragon: Demography, Development, and the Environment in Tibetan Areas.

Winkler, D. 2008b. Yartsa Gunbu (Cordyceps sinensis) and the fungal commodification of the rural economy in Tibet AR. Economic Botany. 62 (3): 291-305.

Zang, M. and Kinjo, N. 1998. Notes on the Alpine Cordyceps of China and nearby nations. Mycotaxon. 66: 215-229.

Zhao, C. S., Yin, W. T., and Wang, J. Y. 2002. CordyMax Cs-4 improves glucose metabolism and increases insulin sensitivity in normal rats. Journal of Alternative and Complementary Medicine. 8 (3): 309-314. 